\title{
Panel discussion section D
}

\section{Chair: Charles R. Cowley}

\author{
SeCtion ORganizer \& KeY-Note SPEAKer: G. Michaud \\ Invited SPEAKERs: G. Alecian, F. LeBlanc \\ Contribution speakers: J. Krtička, R. Monier, O. Richard
}

\section{Discussion}

NoELS: What are the uncertainties in the diffusion velocities? What can we do if we would like to multiply or divide it by something? How can we play with that? What would be your best guess?

Michaud: The atomic diffusion coefficients have been confirmed by different methods one of which is by numerical simulations. They should be relatively accurate. I would think a factor of 1.3 uncertainty is reasonnable. The largest uncertainties are probably from the thermal diffusion coefficients, which are not, however dominant in these stars in general. They play a larger role when elements are highly ionized and so in central regions of stars.

Thermal diffusion is caused by an integration of the interaction of say Fe with the up going and down going protons. In their random motions the protons moving upwards have slightly larger energy than those moving downwards because the former come from higher $T$ regions. The net flux of up going and down going protons is of course 0.0 at equilibrium. It is the energy variation of the scattering cross section that causes thermal diffusion. This crosssection is dominated by small far away collisions. They are affected by Debye shielding which is energy dependent. In the classical Chapman and Cowling expression for thermal diffusion that dependence was not included. They are largely overestimated and should not be used. The Paquette et al. (1986, ApJS, 61, 177) expressions for thermal diffusion are more accurate but they remain more uncertain than the atomic diffusion coefficients. One could have very large diffusion in the central regions of stars due to thermal diffusion if one used the low density limits of the thermal diffusion coefficients as used by Aller \& Chapman (1960, ApJ, 132, 461), for instance, but these are clearly wrong and should not be used. The thermal diffusion coefficients, are very difficult to simulate precisely from first principles and in so far as I know, no statistical physicist is trying to improve the thermal diffusion coefficients we currently have although I have often mentioned the problem. For more details see Michaud (1991, Ann. Phys. (Paris), $16,481)$.

Alecian: There are some other sources of uncertainties. For instance, the turbulent diffusion coefficients are not well known. This makes it difficult to correctly describe the transition between convection zones and radiative zones. On the other hand, there are still some uncertainties about radiative accelerations.

Piskunov: At which point in your calculations do you actually recalculate the ionization equilibrium? Is that during the abundance adjustment or during the temperature correction step? 
LEBLANC: During the temperature correction step.

Piskunov: How much effort would it take to convert the results by Michaud into some kind of tables and then incorporate them in your models?

LEBLANC: I think it would be probably quite complicated. Michaud is working on the same stars as I, but he needs our results as outer boundary conditions. For PHOENIX, of course, we could use pretabulated tables, but in the atmosphere, with blends, it could also be dangerous.

Alecian: The Vienna group is presently preparing a new code which is a paralellized version of the Kurucz Atlas12 model (see Bischof 2005, These Proceedings, AP1). Presently the model atmosphere is an input of our code for the accelerations. A next step will consist in joining both the codes (the one for the accelerations and the other one for the model atmosphere).

LANDSTREeT: Both Monier and Hill find that, even among rapidly rotating $\left(v_{\text {eq }} \geqslant 100\right.$ $\mathrm{km} \mathrm{s}^{-1}$ ) A stars, abundance anomalies exist. If turbulent mixing increases with rotation velocity, as some models predict, how can these anomalies be understood? (Alternatively, one should perhaps try to understand both A and Am stars at the same time.)

MiCHAUD: In the evolutionary model calculations including turbulent diffusion which I duscussed we are not considering a potential dependence of turbulent transport coefficients on rotation velocity. We are using abundance anomalies to constrain turbulent transport coefficients. In a second step the results may be used to constrain meridional circulation, or other sources of instability. In earlier work, Charbonneau \& Michaud (1991, ApJ, 370, 693) had concluded that some overabundances persisted above $100 \mathrm{~km} \mathrm{~s}^{-1}$ when atomic diffusion competed with meridional circulation. They were smaller than below that limit. That was in static models but I would expect the same result to hold in evolutionary models.

Now, in evolutionary models described by Talon and Richard where turbulence is effectively coupled with rotation, the results are not very encouraging in this case with the coefficients used. Many of the coefficients of those models, and presumably the turbulent transport coefficients, are increasing as the square of the rotational velocity. That would mean a relatively rapid decrease of the anomalies as you go beyond about $100 \mathrm{~km} \mathrm{~s}^{-1}$ but not necessarily their disappearance. On the other hand one has to be careful, mass loss could also be involved at the same time.

TALON: Rotational mixing according to the simplest model of meridional circulation gives too strong mixing. It is even worse when you consider that the Eddington-Sweet timescale for stars with rotation velocities below $50 \mathrm{~km} \mathrm{~s}^{-1}$ is as long as the Main Sequence lifetime. However this long timescale implies that the slow rotators have stronger differential rotation, and leads to turbulent diffusion coefficients that do not scale in $\Omega^{2}$ as expected for solid body rotation but are rather much more similar for various velocities (below $100 \mathrm{~km} \mathrm{~s}^{-1}$ ). There remains the problem that turbulence is at least a factor 100 too strong. This could be due to several neglected features namely the stabilizing effect of the mean molecular weight gradient, the erosion of vertical turbulence by horizontal turbulence and/or a cutoff effect due to radiative viscosity. If we find a self consistent model for A and Am stars, we should go back and look at the Geneva group's calculations of abundance anomalies in $\mathrm{O}$ stars to see if the modifications introduced in the model still permit one to explain the observations. 
VAuclair: With Sylvie Théado (2003, ApJ, 587, 777) we have computed the coupling effect of helium diffusion and rotation-induced mixing. The induced $\mu$-gradient has a feed-back effect on the mixing which is not trivial. It depends strongly on the ratio of the horizontal to the vertical diffusion coefficient. In any case there is no single scaling between the effective diffusion coefficient and the rotation velocity.

NoELS: What was the age range of the galactic clusters you analyzed? It would be very interesting to sort out a dependence of the peculiarities in A stars as a function of time by comparing them from young clusters to older ones.

Monier: The clusters range in ages from 45 Myrs to 700 Myrs. The search for a putative dependence of abundances versus age is indeed one of the ultimate goals of this project.

Griffin: The third magnitude binary o Leo consists of 2 Am-like stars, both having classical Am-type abundances. The secondary is indeed an A star, but the primary is evolving. It is crossing the Hertzsprung gap and has $T \approx 6100 \mathrm{~K}$. Does the persistance of the Am phenomenom in such a (relatively speaking) cool giant influence your interpretation of the effects of diffusion? The range of anomalies is just the same.

Michaud: There are still effects of diffusion in the cooler stars but they are much smaller. Diffusion could not explain the abundance anomalies by the same factor in the Sun and in the $7000 \mathrm{~K}$ stars. It is completely excluded. However, when you look at a star cooling on the subgiant branch, the situation is a little different. In Fig. 14 of Richard et al. (2001, ApJ, 558, 377) you may see that abundance anomalies disappear only at $\log g \sim 3.2$ as a $2.5 M_{\odot}$ star cools. We also have figures for the evolution as a function of $T_{\text {eff }}$ (unpublished) and in the same star the disappearance of the anomalies is at 6000 $\mathrm{K}$.

YILDIZ: It is generally adopted that the elements are following the magnetic field lines during the diffusion process. But, I remember a paper from 1980s in which it is found that the equatorial region of young stars, the elements are moving upward crossing the field lines. Can you conclude that how this can be?

Alecian: As I explained in my talk, horizontal diffusion is not the dominant process in element stratifications because of large time scales involved. It is the angular dependence of the vertical component of the diffusion velocity which determines the abundance patches at the surface of magnetic Ap stars. Magnetic fields reduces the diffusion velocity of ions, but not completely. On another hand, elements can often diffuse across magnetic lines because of the contribution of neutral states.

WADE: What are the impediments to developing a coupled, time-dependent, self-consistent model of the interior and atmosphere including diffusion?

LEBLAnC: The first thing are timescales. In the atmospheres the timescales are very short as compared to the interior. So we would need a longer calculation time at least a factor 1000. Also in the atmosophere we need to calculate the radiative field differently than in the interior. In the interior we use the diffusion approximation. While in the atmosphere we absolutly need the detailed radiative field for a large number of points.

Michaud: To properly predict anomalies, one would like to couple atmosphere and mass loss models to evolutionary models. It is probably possible to make the assumption 
that the atmospheric part has come to some equilbrium with what is coming from below. Simply modify that atmospheric equilbrium abundance progressively.

At the moment what we do for the atmospheres in the evolutionary model is very approximate. But if you wish to evaluate the possibility of selective mass loss in particular, we have to be much more sophisticated than we currently are in the calculations of the atmospheric region.

WADE: But what is the solution? Is it a question of bigger and faster computer?

Michaud: I think first one must develop the proper selective mass loss models. I do not think that has yet been done to an acceptable degree of accuracy and then we can couple them to the evolutionary models. But those models have to be developed. It is a lot of software development.

BudAJ: I just want to address Wade's question. A possible way to couple these interior calculations with the radiative transfer envelopes follows. Calculations in the envelope can correlate the boundary condition in the sense that we would know the abundance gradient at the bottom of the atmosphere and this would provide us with the flux of the element at that point and the condition in the atmosphere would not be a zero diffusion velocity but a constant particle flux. This would couple the atmosphere with the interior. So we would just need two numbers. The concentration and gradient at the bottom of the atmosphere.

PAUnzen: We heard a lot about the stars with overabundances. I am interested in stars with underabundaces, which are the $\lambda$ Bootis stars. A few years ago there were some papers concluding that diffusion together with mass loss can produce underabundances in 1 Gyr or so. We heard a great deal about new calculations. Is there any news about what underabundances can be generated with diffusion together with mass loss? At earlier stages of the stellar evolution? Is it possible to produce underabundances on timescales smaller than 1 Gyr?

COWLEY: That is actually a question I have been interested in asking as well. What is the current standing from the theoretical point of view of stars with underabundances, the so-called $\lambda$ Boo stars, which form apparently a very inhomogeneous group. Can we understand now, young objects with underabundances?

Michaud: The diffusion models that have been proposed for $\lambda$ Boo stars, where there is radiative diffusion and undifferentiated mass loss (Michaud \& Charland 1986, ApJ, $311,326)$ required very large timescales, longer than some of the clusters where some $\lambda$ Boo stars had been observed. For that reason atomic diffusion does not appear as the most likely explantion for that. A differentiated mass loss as has been discussed today might succeed, in combination with atomic diffusion, but that requires a credible model of the differentiated mass loss. Separation would then occur partly in the wind. So that is something that should be investigated, but the explanation for these stars could not be only atomic diffusion in the stellar interior. That requires too long timescales. As an alternative, accretion has been investigated with some success by Turcotte \& Charbonneau (1993, ApJ, 413, 376). 